\title{
Simultaneous multi-frequency flux monitoring of $0716+714$ with KVN 21-m radio telescopes
}

\author{
Jee Won Lee ${ }^{* \dagger}$ \\ Korean VLBI network, Korea Astronomy and Space Science Institute \\ Dept. of Astronomy and Space Science, Kyung Hee University, Korea \\ E-mail: jwlee78@kasi.re.kr

\section{Bong Won Sohn, Do-Young Byun, Jeong Ae Lee} \\ Korean VLBI network, Korea Astronomy and Space Science Institute
}

\section{Sungsoo S. Kim}

Dept. of Astronomy and Space Science, Kyung Hee University, Korea E-mail: sskimekhu.ac.kr

\begin{abstract}
We observed the BL Lac object $0716+714$ at 22 and $43 \mathrm{GHz}$ simultaneously to investigate its radio flux variability. This radio source is well known as an intraday variable (IDV) source which is characterized by rapid flux variations on time scale of a day or less. The observations were performed over three seasons with Korean VLBI Network (KVN) radio telescopes by using single- dish observation mode. The first season consists of four epochs between J.D.=2455177 and J.D.=2455364 and each epoch was performed for 24 hours over 3-8 days to search for IDV of $0716+714$. But significant fast variations such as IDV was not detected at both frequencies in the first season and only monotonic increases and decreases of flux are detected. In the second and third season, we obtained data a three or four-day interval from J.D. $=2455511$ to J.D. $=2455747$, and from J.D.=2455871 to J.D.=2456055 to search for longer term variability of 0716+714. Here, we present results obtained from multi-frequency observations of $0716+714$ over three seasons.
\end{abstract}

11th European VLBI Network Symposium \& Users Meeting,

October 9-12, 2012

Bordeaux, France

* Speaker.

${ }^{\dagger}$ A footnote may follow. 


\begin{tabular}{cccc}
\hline Season & Date & J.D.-2450000 & Observatory \\
\hline \hline 1 & Dec. 11, 2009 - Dec. 15, 2009 & $5177.27-5181.41$ & Yonsei \\
& Jan. 4, 2010 - Jan. 11, 2010 & $5201.54-5208.02$ & Yonsei \\
& Jan. 27, 2010 - Jan. 30, 2010 & $5224.30-52227.10$ & Yonsei \\
& Jun. 14, 2010 - Jun. 16, 2010 & $5362.36-5364.21$ & Yonsei \\
\hline 2 & Nov. 5, 2010 - Jun. 29, 2011 & $5511.02-5747.02$ & Yonsei, Ulsan \\
\hline 3 & Oct. 18, 2011 - Apr. 29, 2012 & $5871.04-6055.04$ & Yonsei, Ulsan, Tamna \\
\hline
\end{tabular}

Table 1: Observation date

\section{Introduction}

Active galactic nuclei (AGN) with flat-spectrum $\left(\mathrm{S}_{v} \propto v^{-\alpha} ; \alpha>-0.5\right)$ such as blazars and quasars have been well known to have significant flux variations on time scales of days or less across the whole electromagnetic spectrum ([1], [2]). In particular, the flux variations in the radio regime are more important to understand the emission mechanism of the AGN jet. Intraday variations (IDV) have been found in a large fraction ( 20-30\%) of flat-spectrum radio sources ([1], [3]). "Type II" IDV sources show variations in flux with time scale of $<0.5-2$ days and their amplitudes change up to $30 \%$ in the radio bands. In contrast, the flux of "Type I" IDV sources changes monotonically on time scales of $>2$ days. The IDV phenomenon is interpreted as a model of intrinsic variation (shock-in-jet model) as well as a model of extrinsic variation (interstellar scintillation). However, interstellar scintillation mainly occurs at $\mathrm{cm}$ wavelengths. If the fast variations are intrinsic to the source the flux variations can be used to estimate the angular size of the emitting region with light-travel-time argument. Furthermore, the fast variations are related to the high apparent brightness temperature $T_{B} \geq 10^{18} \mathrm{~K}$ largely exceeding Inverse-Compton (IC) limit of $10^{12} \mathrm{~K}$ ([4]). In order to bring down the brightness temperature to the IC limit, the high relativistic Dopplerboosting factors as high as $>100$ would be required. However, such high doppler factors have never been detected in AGN jets with VLBI observations until now ([5], [6]). Therefore intrinsic model in order to explain the fast variations such as IDV is still unclear.

In order to study a physical origin of $0716+714$, we performed simultaneous multi-frequency monitoring of $0716+714$ with $\mathrm{KVN} 21-\mathrm{m}$ radio telescopes at $22 \mathrm{GHz}$ and $43 \mathrm{GHz}$. The BL Lac object 0716+714 is the most studied rapid variable source at all wavelengths ([7], [8], [9], [11], [12], [13], [14]). In particular, drastic variations within 2 days with large amplitude of up to 10 percent had been detected at cm-wavelengths by [1] and [3]. Kraus also observed $20 \%$ peak-topeak amplitude fast flux variations within 24 hours at $9 \mathrm{~mm}$ in 1998 ([9]).

\section{The observations and data reduction}

The simultaneous flux monitoring of $0716+714$ at $22 \mathrm{GHz}$ and $43 \mathrm{GHz}$ was performed with KVN 21-m radio telescopes located in the three observatories which are Yonsei, Ulsan and Tamna university in Korea. We repeatedly monitored $0716+714$ over three seasons. Detailed observation date and participating observatories are summarized in Table 1. The season 1 consists of four epochs and each epoch was observed continuously over 3-8 days to search for intraday variability in flux of $0716+714$. For the season 2 and the season 3 which ranged from J.D.= 2455511.02 - 

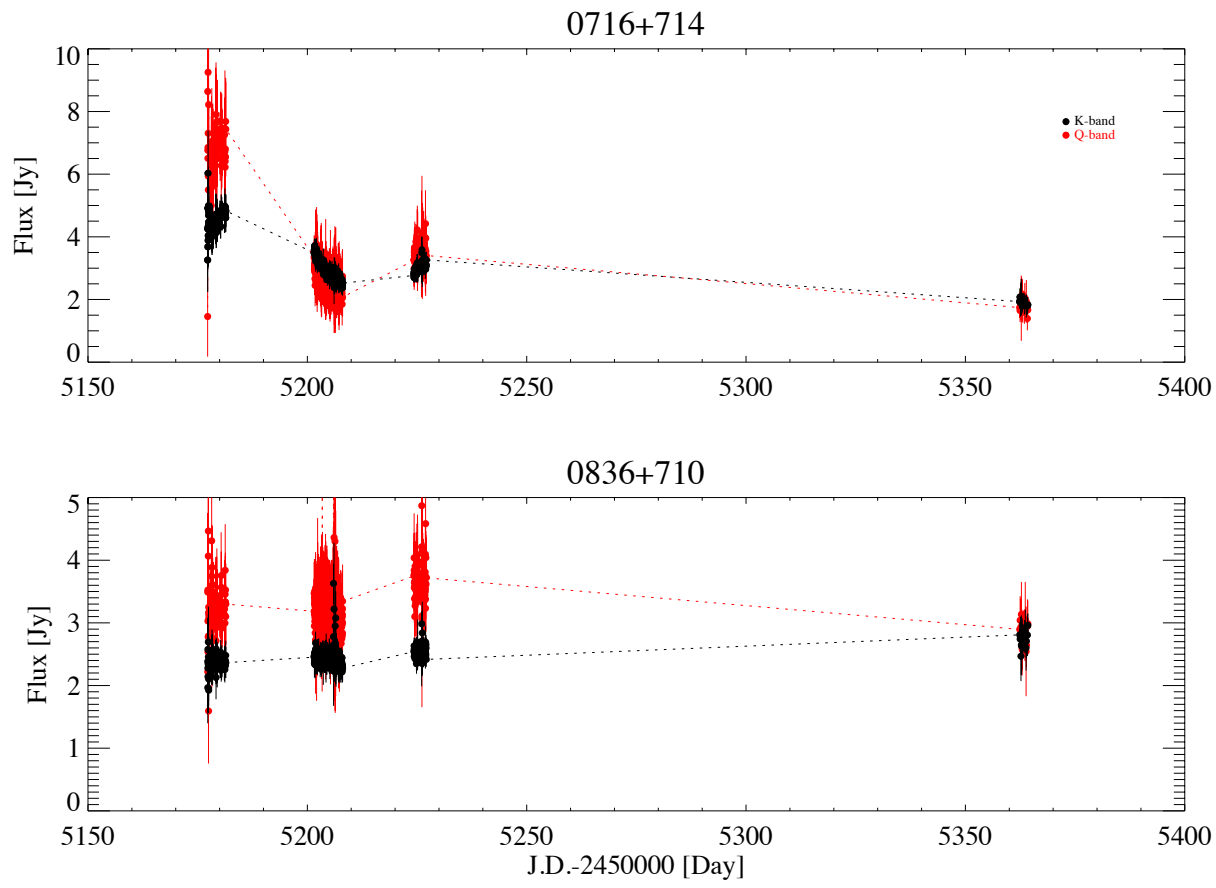

Figure 1: The light curve of $0716+714$ and $0836+710$ (calibrator) obtained at $22 \mathrm{GHz}$ (black symbol) and $43 \mathrm{GHz}$ (red symbol) in all the epochs of season 1.

2455747.02 and J.D.= $2455871.04-2456066.04$ respectively, we obtained a data point generally every three or four days except for bad weather condition. Furthermore, when $0716+714$ was in high-state, daily observations were performed from J.D. 2455725 to 2455747 in season 2. The target source $0716+714$ and a secondary calibrator $0836+710$ were observed alternately with a dense time-sampling. The source 3C286 was used as a flux calibrator and measured every 1-2 hours in all seasons. The observations of all seasons were made at 21.7 and $42.4 \mathrm{GHz}$ simultaneously and the total bandwidth was $512 \mathrm{MHz}$. Detailed specifications of KVN radio telescope were publishied by [10]. All the measurements of the flux were performed by cross-scan mode in azimuth and elevation direction through the source position [1]. The number of cross-scans for an individual source were composed of 10 sub-scans with integration times of 40 seconds and 20 seconds at 22 and $43 \mathrm{GHz}$ respectively. The data reduction procedure consists in the following steps: 1) baseline fitting 2) gauss fitting 3) removing bad scans 4) pointing-offset correction 5) flux conversion.

\section{Results}

\subsection{Season 1}

Figure 1 shows light curves of $0716+714$ obtained at 22 and $43 \mathrm{GHz}$ with KVN 21 -m radio telescopes in season 1. For a direct comparison, the secondary calibrator $0836+710$ flux is also displayed. In this observing season, intensive flux monitoring for 24 hours over four epochs was performed to investigate intraday variability of $0716+714$. However, the typical "Type II" IDV behaviour was not detected at the two observing frequencies at any epochs. Only monotonic increase 

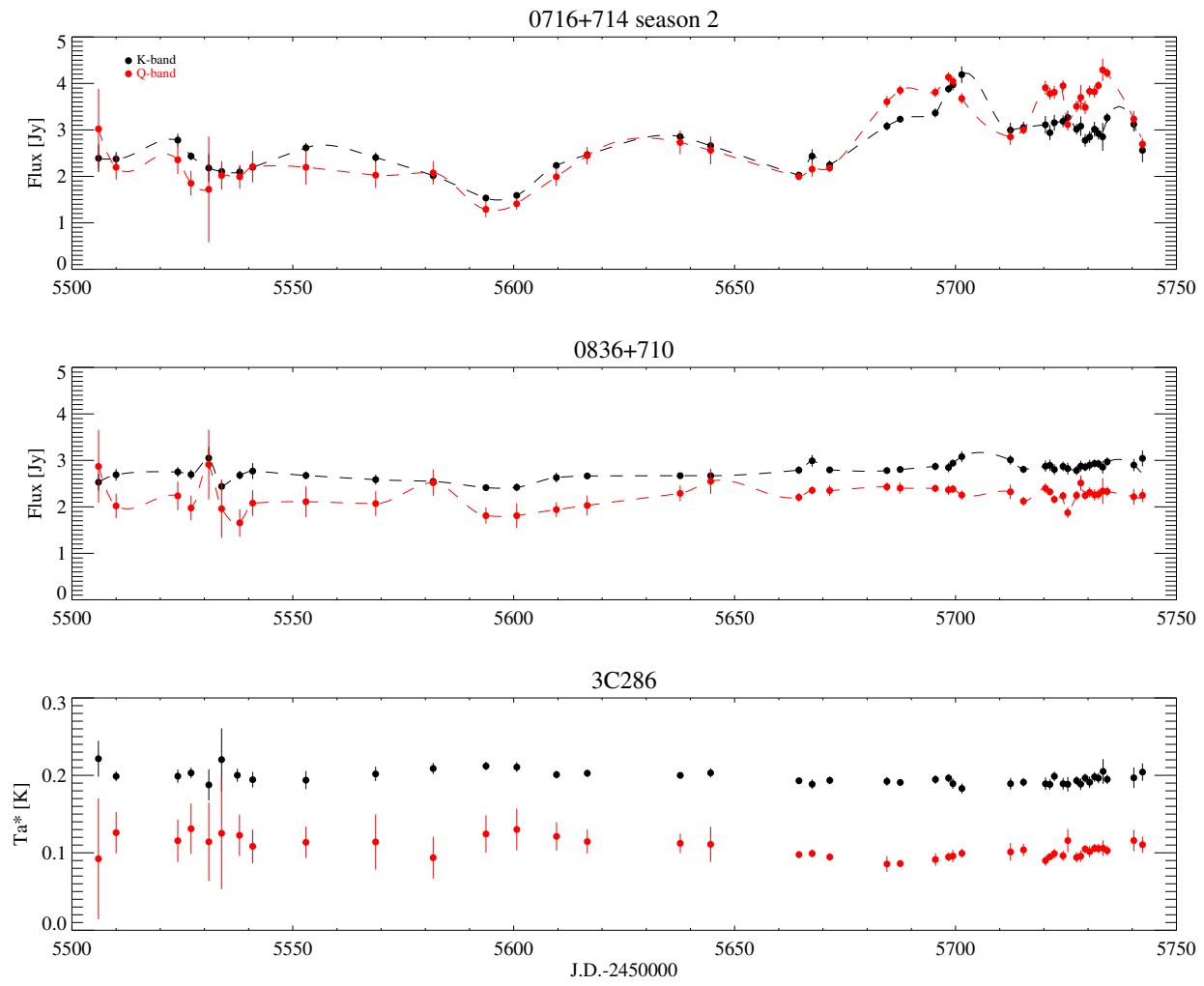

Figure 2: The light curve of 0716+714 (top panel) at 22 and $43 \mathrm{GHz}$ in season 2 . For a direct comparison, the secondary calibrator 0836+710 (middle panel) and flux calibrator 3C286 (bottom pannel) are plotted. 22 $\mathrm{GHz}$ data are displayed as black dots, and $43 \mathrm{GHz}$ data as red dots.

and decrease of the flux over a time range of several days were detected in all epochs. In particular, the epoch 1 (from J.D.=2455177.27 to J.D.=2455181.41) shows the most high state of 0716+714. The flux are $4.46 \mathrm{Jy}$ at $22 \mathrm{GHz}$ and $6.76 \mathrm{Jy}$ at $43 \mathrm{GHz}$. These flux of epoch 1 are about 2-3 times brighter than the flux detected at epoch 4 (from J.D.=2455362.36 to 2455364.21). 0716+714 shows obvious inverted spectrum in epoch 1.

\subsection{Season 2 and Season 3}

The season 2 and season 3 were performed to study relatively longer-term variability of $0716+714$ on a time scale of a month. We plot the light curves obtained simultaneously at 22 and $43 \mathrm{GHz}$ in season 2 (from J.D.=2455511 to J.D.=2455747) and season 3 (J.D.=2455871 to 2456055). Significant flux variations from $0716+714$ were detected at the two frequencies over season 2 when compared with the stationary flux of the secondary calibrator $0836+710$ and flux calibrator 3C286 (see figure 2). On the other hand, the light curve of $0716+714$ for season 3 shows relatively slow variations and low-state at the two frequencies. We used then statistical method such as the modulation index $m$, a reduced chi-square test $\chi_{r}^{2}$-test and a variability amplitude $Y$ study in order to quantify the amount of the variability in light curves. These methods are described in detail in [1], [3] and [14]. The results of these variability analysis are summarized in table 2. To further quantify the characteristic time scale of variability of $0716+714$, we used the structure 
Table 2: The maximum, minimum, and mean flux along with the modulation index, the variability index, the reduced chi-square at 22 and $43 \mathrm{GHz}$.

\begin{tabular}{cccccccccc}
\hline \hline Season & Source & $\begin{array}{c}\text { Frequency } \\
{[\mathrm{GHz}]}\end{array}$ & Data points & $\begin{array}{c}S_{\text {mean }} \\
{[\mathrm{Jy}]}\end{array}$ & $\begin{array}{c}S_{\min } \\
{[\mathrm{Jy}]}\end{array}$ & $\begin{array}{c}S_{\max } \\
{[\mathrm{Jy}]}\end{array}$ & $\begin{array}{c}m \\
{[\%]}\end{array}$ & $\begin{array}{c}Y \\
{[\%]}\end{array}$ & $\chi_{r}^{2}$ \\
\hline 2 & $0716+714$ & 22 & 43 & 2.76 & 1.53 & 4.19 & 20.81 & 59.83 & 118.7 \\
& & 43 & 43 & 2.95 & 1.29 & 4.29 & 29.89 & 83.32 & 407.4 \\
& \multirow{2}{*}{$0836+710$} & 22 & 43 & 2.79 & 2.41 & 3.08 & 5.96 & & 10.3 \\
& & 43 & 43 & 2.24 & 1.66 & 2.91 & 11.06 & & 1.5 \\
\hline 3 & $0716+714$ & 22 & 28 & 1.80 & 0.91 & 3.43 & 32.58 & 96.56 & 255.5 \\
& & 43 & 28 & 1.70 & 0.92 & 2.75 & 27.15 & 75.90 & 4.5 \\
& \multirow{2}{*}{$0836+710$} & 22 & 28 & 2.82 & 2.64 & 3.17 & 5.06 & & 0.39 \\
& & 43 & 28 & 1.697 & 1.29 & 1.96 & 9.85 & & 0.015 \\
\hline
\end{tabular}

$0716+714$ season 3

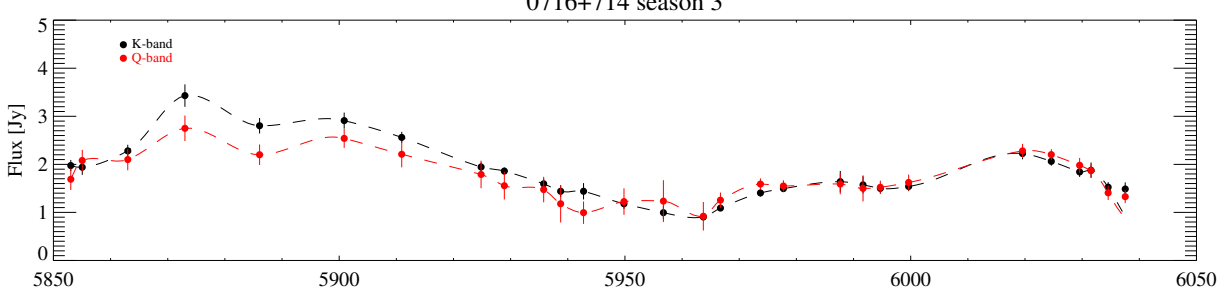

$0836+710$

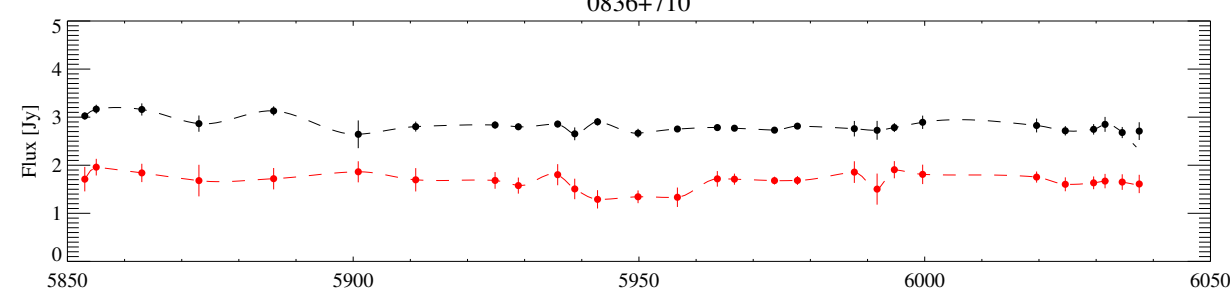

$3 \mathrm{C} 286$

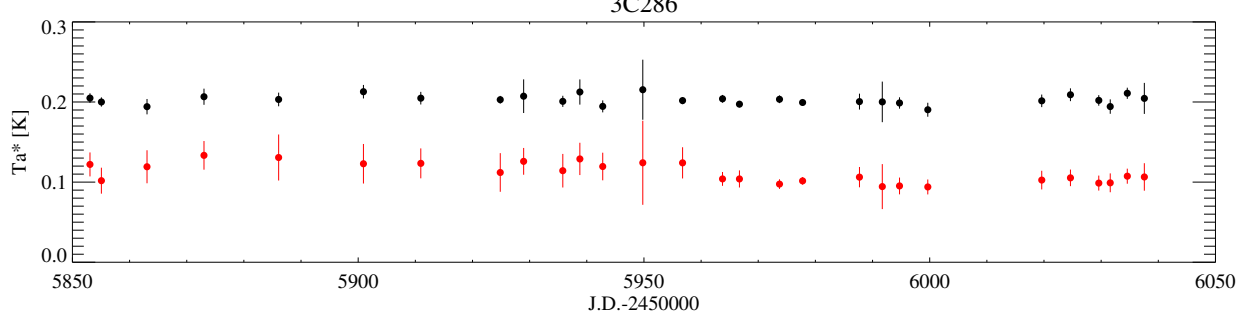

Figure 3: The light curve of $0716+714$ obtained at 22 and $43 \mathrm{GHz}$ in season 3. The secondary calibrator $0836+710$ and the flux calibrator 3C286 are also displayed. $22 \mathrm{GHz}$ dat are displayed as black dots, and 43 $\mathrm{GHz}$ data as red dots. 

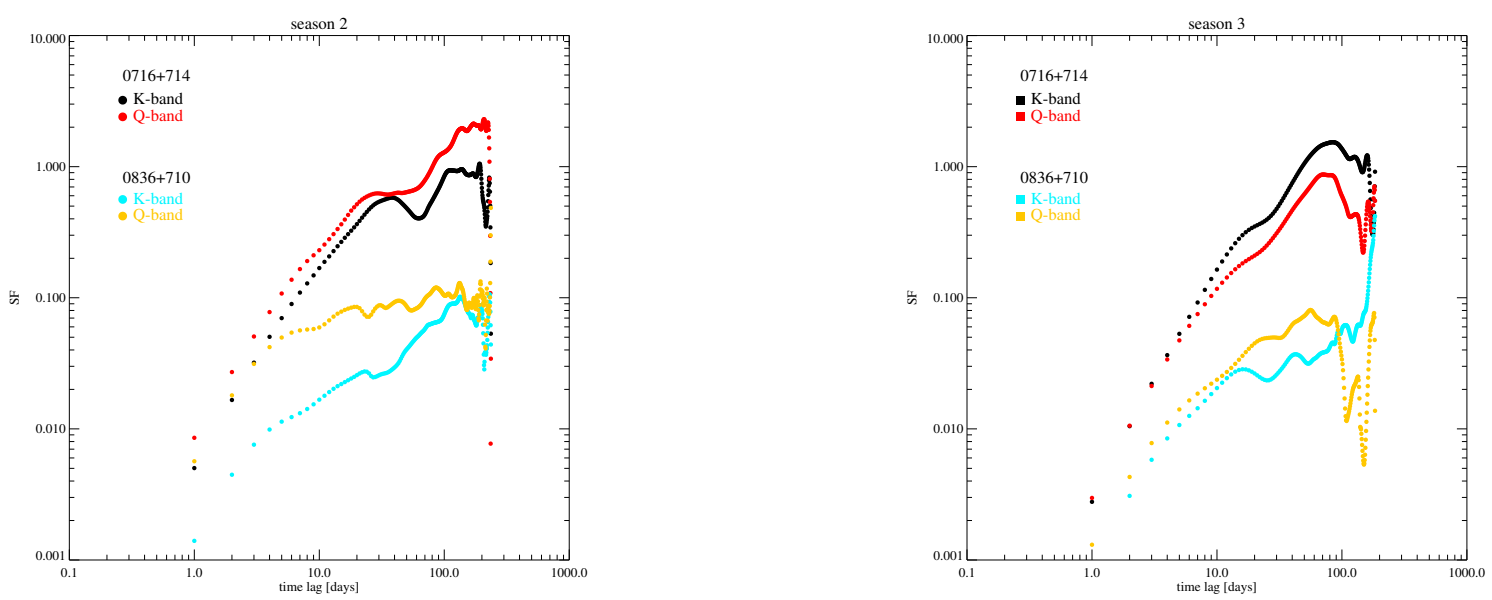

Figure 4: The plot of structure functions (SF) versus time lag of $0716+714$ and $0836+710$ at 22 and $43 \mathrm{GHz}$. The left-panel shows the SF in season2, and the right-panel the SF in season3.

function analysis (SF). The structure function is defined by [15]. Figure 4 shows results of the SF versus time lag from $0716+714$ and $0836+710$ for each season. In this figure, when compared with the calibrator $0836+710$, the steeper slope and larger first maximum of the SF of $0716+714$ implies a larger amplitude of variability. The first maximum of the SF reflects the characteristic time scale of the variations. Therefore we measured a characteristic time scale of $\sim 38$ days at $22 \mathrm{GHz}$ and $\sim 29$ days at $43 \mathrm{GHz}$ in season 2. However, in season 3, a precise determination of a characteristic time scale was difficult because of the absence of distinct first maximum of the SF.

\section{References}

[1] Heeschen, D. S., Krichbaum, T., Schalinski, C. J., \& Witzel, A., AJ, 94, 1493 (1987)

[2] Wagner, S. J., \& Witzel, A., ARA\&A, 33, 163 (1995)

[3] Quirrenbach, A., Witzel, A., Kirchbaum, T. P., et al., A\&A, 258, 279 (1992)

[4] Kellermann, K. I., Pauliny-Toth, I. I. K., \& Williams, P. J. S., ApJ, 157, 1 (1969)

[5] Witzel, A., Schalinski, C. J., Johnston, K. J., et al.,A\&A, 206, 245 (1998)

[6] Ghisellini, G., Padovani, P., Celotti, A., \& Maraschi, L., ApJ, 407, 65 (1993)

[7] Wagner, S. J., Witzel, A., Krichbaum, T. P., et al., A\&A, 271, 344 (1993)

[8] Wagner, S. J., Witzel, A., Heidt, J., et al., AJ, 111, 2187 (1996)

[9] Kraus, A., Krichbaum, T. P., Wegner, R., et al., $A \& A, 4$ 401, 161 (2003)

[10] Lee, S.-S., Byun, D.-Y., Oh, C. S., et al. 2011, PASP, 123, 1398 (2011)

[11] Raiteri, C. M., Villata, M., Tosti, G., et al., A\&A, 402, 151 (2003)

[12] Ostorero, L., Wagner, S. J., Gracia, J., et al., $A \& A, 4$ 451, 797 (2006)

[13] Agudo, I., Krichbaum, T. P., Ungerechts, H., et al., $A \& A$, 456, 117 (2006)

[14] Fuhrmann, L., Krichbaum, T. P., Witzel, A., et al., A\&A, 490, 1019 (2008)

[15] Simonetti, J. H., Cordes, J. M., \& Heeschen, D. S. 1985, ApJ, 296, 46 (1985) 